\title{
Some results on a general iterative method for $k$-strictly pseudo-contractive mappings
}

Jong Soo Jung

Correspondence: jungjs@mail. donga.ac.kr

Department of Mathematics, Dong-A University, Busan 604-714, Korea

\begin{abstract}
Let $H$ be a Hilbert space, $C$ be a closed convex subset of $H$ such that $C \pm C \subset C$, and $T: C \rightarrow H$ be a $k$-strictly pseudo-contractive mapping with $F(T) \neq \varnothing$ for some $0 \leq k$ $<1$. Let $F: C \rightarrow C$ be a $\kappa$-Lipschitzian and $\eta$-strongly monotone operator with $\kappa>0$ and $\eta>0$ and $f: C \rightarrow C$ be a contraction with the contractive constant $\alpha \in(0,1)$. Let $0<\mu<\frac{2 \eta}{\kappa^{21}} 0<\gamma<\frac{\mu\left(\eta-\frac{\mu \kappa^{2}}{2}\right)}{\alpha}=\frac{\tau}{\alpha}$ and $\tau<1$. Let $\left\{\alpha_{n}\right\}$ and $\left\{\beta_{n}\right\}$ be sequences in $(0,1)$. It is proved that under appropriate control conditions on $\left\{\alpha_{n}\right\}$ and $\left\{\beta_{n}\right\}$, the sequence $\left\{x_{n}\right\}$ generated by the iterative scheme $x_{n+1}=\alpha_{n} \gamma f\left(x_{n}\right)+\beta_{n} x_{n}+\left(\left(1-\beta_{n}\right) l-\right.$ $\left.\alpha_{n} \mu F\right) P_{C} S x_{n}$, where $S: C \rightarrow H$ is a mapping defined by $S x=k x+(1-k) T x$ and $P_{C}$ is the metric projection of $H$ onto $C$, converges strongly to $q \in F(T)$, which solves the variational inequality $\langle\mu F q-\gamma(q), q-p\rangle \leq 0$ for $p \in F(T)$.

MSC: 47H09, 47H05, 47H10, 47J25, 49M05
\end{abstract}

Keywords: Iterative schemes, $k$-strictly pseudo-contractive mapping, Nonexpansive mapping, Fixed points, Contraction, $k$-Lipschitzian, $\eta$-strongly monotone operator, Variational inequality, Hilbert space

\section{Introduction}

Let $H$ be a real Hilbert space and $C$ be a nonempty closed convex subset of $H$. Recall that a mapping $f: C \rightarrow C$ is a contraction on $C$ if there exists a constant $\alpha \in(0,1)$ such that $\|f(x)-f(y)\| \leq \alpha|| x-y \|, x, y \in C$. A mapping $T: C \rightarrow H$ is said to be $k$-strictly pseudo-contractive if there exists a constant $k \in[0,1)$ such that

$$
\|T x-T y\|^{2} \leq\|x-y\|^{2}+k\|(I-T) x-(I-T) y\|^{2}, \quad x, y \in C,
$$

and $F(T)$ denote the set of fixed points of the mapping $T$; that is, $F(T)=\{x \in C$ : $T x=x\}$.

Note that the class of $k$-strictly pseudo-contractions includes the class of non-expansive mappings $T$ on $C$ (that is, $\|T x-T y\| \leq\|x-y\|, x, y \in C$ ) as a subclass. That is, $T$ is nonexpansive if and only if $T$ is 0 -strictly pseudo-contractive. The mapping $T$ is also said to be pseudo-contractive if $k=1$ and $T$ is said to be strongly pseudo-contractive if there exists a constant $\lambda \in(0,1)$ such that $T-\lambda I$ is pseudo-contractive. Clearly, the class of $k$-strictly pseudo-contractive mappings falls into the one between classes of nonexpansive mappings and pseudo-contractive mappings. Also we remark that the class of strongly pseudo-contractive mappings is independent of the class of $k$-strictly pseudo-contractive mappings (see [1-3]). The class of pseudo-contraction is one of the

\section{SpringerOpen ${ }^{\circ}$}

(C) 2011 Jung; licensee Springer. This is an Open Access article distributed under the terms of the Creative Commons Attribution License (http://creativecommons.org/licenses/by/2.0), which permits unrestricted use, distribution, and reproduction in any medium, provided the original work is properly cited. 
most important classes of mappings among nonlinear mappings. Recently, many authors have been devoting the studies on the problems of finding fixed points for pseudo-contractions, see, for example, [4-7] and references therein.

For nonexpansive mappings, one recent way to study them is to construct the iterative scheme, the so-called viscosity iteration method: more precisely, for a nonexpansive mapping $T$, a contraction $f$ with the contractive constant $\alpha \in(0,1)$, and $\alpha_{n} \in(0,1)$,

$$
x_{n+1}=\alpha_{n} f\left(x_{n}\right)+\left(1-\alpha_{n}\right) T x_{n}, \quad n \geq 0 .
$$

This iterative scheme was first introduced by Moudafi [8].

In particular, under the control conditions on $\left\{\alpha_{n}\right\}$

(C1) $\lim _{n \rightarrow \infty} \alpha_{n}=0$;

(C2) $\Sigma_{n=0}^{\infty} \alpha_{n}=\infty$;

(C3) $\Sigma_{n=0}^{\infty}\left|\alpha_{n+1}-\alpha_{n}\right|<\infty$; or,

(C4) $\lim _{n \rightarrow \infty} \frac{\alpha_{n+1}}{\alpha_{n}}=1$,

$\mathrm{Xu}[9]$ proved that the sequence $\left\{x_{n}\right\}$ generated by (1.1) converges strongly to a fixed point $q$ of $T$, which is the unique solution of the following variational inequality:

$$
\langle q-f(q), q-p\rangle \leq 0, \quad p \in F(T) .
$$

Recall that an operator $A$ is strongly positive on $H$ if there exists a constant $\bar{\gamma}>0$ with the property:

$$
\langle A x, x\rangle \geq \bar{\gamma}\|x\|^{2}, \quad x \in H .
$$

In 2006, as the viscosity approximation method, Marino and $\mathrm{Xu}$ [10] considered the following iterative method: for a strongly positive bounded linear operator $A$ on $H$ with constant $\bar{\gamma}>0$, a nonexpansive mapping $T$ on $H$, a contraction $f: H \rightarrow H$ with the contractive constant $\alpha \in(0,1),\left\{\alpha_{n}\right\} \subset(0,1)$ and $\gamma>0$,

$$
x_{n+1}=\left(I-\alpha_{n} A\right) T x_{n}+\alpha_{n} \gamma f\left(x_{n}\right), \quad n \geq 0 .
$$

They proved that if the sequence $\left\{\alpha_{n}\right\}$ satisfies the conditions (C1), (C2), and (C3) (or $(\mathrm{C} 1),(\mathrm{C} 2)$, and $(\mathrm{C} 4))$, then the sequence $\left\{x_{n}\right\}$ generated by (1.2) converges strongly to the unique solution of the variational inequality

$$
\left\langle(A-\gamma f) x^{*}, x-x^{*}\right\rangle \geq 0, \quad x \in F(T),
$$

which is the optimality condition for the minimization problem

$$
\min _{x \in F(T)} \frac{1}{2}\langle A x, x\rangle-h(x),
$$

where $h$ is a potential function for $\gamma f$.

In 2010, in order to improve the corresponding results of Cho et al. [5] as well as Marino and $\mathrm{Xu}$ [10] by removing the condition (C3), Jung [6] studied the following composite iterative scheme for the class of $k$-strictly pseudo-contractive mappings.

Theorem J. Let $H$ be a Hilbert space, $C$ be a closed convex subset of $H$ such that $C \pm$ $C \subset C, T: C \rightarrow H$ be a $k$-strictly pseudo-contractive mapping with $F(T) \neq \varnothing$, for some $0 \leq k<1$. Let $A$ be a strongly positive bounded linear operator on $C$ with constant $\bar{\gamma} \in(0,1)$ and $f: C \rightarrow C$ be a contraction with the contractive constant $\alpha \in(0,1)$ such that $0<\gamma<\frac{\bar{\gamma}}{\alpha}$. Let $\left\{\alpha_{n}\right\}$ and $\left\{\beta_{n}\right\}$ be sequences in $(0,1)$ satisfying the conditions (C1), 
(C2) and the condition $0<\lim \inf _{n \rightarrow \infty} \beta_{n} \leq \lim \sup _{n \rightarrow \infty} \beta_{n}<1$. Let $\left\{x_{n}\right\}$ be a sequence in $C$ generated by

$$
\left\{\begin{array}{l}
x_{0} \in C \\
y_{n}=\beta_{n} x_{n}+\left(1-\beta_{n}\right) P_{C} S x_{n}, \\
x_{n+1}=\alpha_{n} \gamma f\left(x_{n}\right)+\left(I-\alpha_{n} A\right) y_{n}, \quad n \geq 0,
\end{array}\right.
$$

where $S: C \rightarrow H$ is a mapping defined by $S x=k x+(1-k) T x$ and $P_{C}$ is the metric projection of $H$ onto $C$. Then $\left\{x_{n}\right\}$ converges strongly to a fixed point $q$ of $T$, which is the unique solution of the following variational inequality related to the linear operator A:

$$
\langle\gamma f(q)-A q, p-q\rangle \leq 0, \quad p \in F(T) .
$$

On the other hand, a mapping $F: H \rightarrow H$ is called $\kappa$-Lipschitzian if there exists a positive constant $\kappa$ such that

$$
\|F x-F y\| \leq \kappa|| x-y \|, \quad x, y \in H .
$$

$F$ is said to be $\eta$-strongly monotone if there exists a positive constant $\eta$ such that

$$
\langle F x-F y, x-y\rangle \geq \eta\|x-y\|^{2}, \quad x, y \in H .
$$

From the definitions, we note that a strongly positive bounded linear operator $A$ is a $\|A\|$-Lipschitzian and $\bar{\gamma}$-strongly monotone operator.

In 2001, Yamada [11] introduced the following hybrid iterative method for solving the variational inequality

$$
x_{n+1}=\left(I-\mu \lambda_{n} F\right) S x_{n}, \quad n \geq 1,
$$

where $F: H \rightarrow H$ is a $\kappa$-Lipschitzian and $\eta$-strongly monotone operator with $\kappa>0$, $\eta>0,0<\mu<\frac{2 \eta}{\kappa^{2}}$ and $S: H \rightarrow H$ is a nonexpansive mapping, and proved that if $\left\{\lambda_{n}\right\}$ satisfies appropriate conditions, then the sequence $\left\{x_{n}\right\}$ generated by (1.5) converges strongly to the unique solution of the variational inequality

$$
\langle F \tilde{x}, x-\tilde{x}\rangle \geq 0, \quad x \in F(S) .
$$

In 2010, by combining the iterative method (1.2) with the Yamada's method (1.5), Tian [12] considered the following general iterative method.

Theorem T1. Let $H$ be a Hilbert space, $F: H \rightarrow H$ be a $\kappa$-Lipschitzian and $\eta$-strongly monotone operator with $\kappa>0$ and $\eta>0$, and $S: H \rightarrow H$ be a nonexpansive mapping with $F(S) \neq \varnothing$. Let $f: H \rightarrow H$ be a contraction with the contractive constant $\alpha$ $\in(0,1)$. Let $0<\mu<\frac{2 \eta}{\kappa^{2}}$ and $0<\gamma<\frac{\mu\left(\eta-\frac{\mu \kappa^{2}}{2}\right)}{\alpha}=\frac{\tau}{\alpha}$. Let $\left\{\alpha_{n}\right\}$ be a sequence in $(0,1)$ satisfying the conditions (C1), (C2) and (C3) (or (C1), (C2) and (C4)). Let $\left\{x_{n}\right\}$ be a sequence in $H$ generated by

$$
\left\{\begin{array}{l}
x_{0} \in H \\
x_{n+1}=\alpha_{n} \gamma f\left(x_{n}\right)+\left(I-\alpha_{n} \mu F\right) S x_{n}, \quad n \geq 0 .
\end{array}\right.
$$

Then $\left\{x_{n}\right\}$ converges strongly to a fixed point $\tilde{x}$ of $S$, which is the unique solution of the following variational inequality related to the operator $F$ :

$$
\langle\mu F \tilde{x}-\gamma f(\tilde{x}), \tilde{x}-z\rangle \leq 0, \quad z \in F(S) .
$$

In this paper, motivated by the above-mentioned results, we consider the following general iterative scheme for strictly pseudo-contractive mapping: for $C$ a closed convex 
subset of $H$ such that $C \pm C \subset C, k$-strictly pseudo-contractive mapping $T: C \rightarrow H$ with $F(T) \neq \varnothing$, a contraction $f: C \rightarrow C$ with the contractive constant $\alpha \in(0,1), \mu>0$ and $\left\{\alpha_{n}\right\},\left\{\beta_{n}\right\} \subset(0,1)$,

$$
\left\{\begin{array}{l}
x_{0} \in C, \\
x_{n+1}=\alpha_{n} \gamma f\left(x_{n}\right)+\beta_{n} x_{n}+\left(\left(1-\beta_{n}\right) I-\alpha_{n} \mu F\right) P_{C} S x_{n}, \quad n \geq 0,
\end{array}\right.
$$

where $S: C \rightarrow H$ is a mapping defined by $S x=k x+(1-k) T x, P_{C}$ is the metric projection of $H$ onto $C$, and $F: C \rightarrow C$ is a $\kappa$-Lipschitzian and $\eta$-strongly monotone operator with $\kappa>0$ and $\eta>0$. Under certain different control conditions on $\left\{\alpha_{n}\right\}$, we establish the strong convergence of the sequence $\left\{x_{n}\right\}$ generated by (IS) to a fixed point of $T$, which is a solution of the variational inequality (1.6) related to the operator $F$. By removing the condition (C3) $\sum_{n=0}^{\infty}\left|\alpha_{n+1}-\alpha_{n}\right|<\infty$ on $\left\{\alpha_{n}\right\}$, the main results improve, develop and complement the corresponding results of Tian [12] as well as Cho et al. [5], Jung [6] and Marino and $\mathrm{Xu}$ [10]. Our results also improve the corresponding results of Halpern [13], Moudafi [8], Wittmann [14] and Xu [9].

\section{Preliminaries and lemmas}

Throughout this paper, when $\left\{x_{n}\right\}$ is a sequence in $E$, then $x_{n} \rightarrow x$ (resp., $x_{n} \rightarrow x$ ) will denote strong (resp., weak) convergence of the sequence $\left\{x_{n}\right\}$ to $x$.

For every point $x \in H$, there exists a unique nearest point in $C$, denoted by $P_{C}(x)$, such that

$$
\left\|x-P_{C}(x)\right\| \leq\|x-y\|
$$

for all $y \in C$. $P_{C}$ is called the metric projection of $H$ onto $C$. It is well known that $P_{C}$ is nonexpansive.

In a Hilbert space $H$, we have

$$
\|x-y\|^{2}=\|x\|^{2}+\|y\|^{2}-2\langle x, y\rangle \text { for } x, y \in H .
$$

It is also well known that $H$ satisfies the Opial condition, that is, for any sequence $\left\{x_{n}\right\}$ with $x_{n} \rightarrow x$, the inequality

$$
\liminf _{n \rightarrow \infty}\left\|x_{n}-x\right\|<\liminf _{n \rightarrow \infty}\left\|x_{n}-y\right\|
$$

holds for every $y \in H$ with $y \neq x$.

We need the following lemmas for the proof of our main results.

Lemma 2.1 [15]. Let $H$ be a Hilbert space and $C$ be a closed convex subset of $H$. If $T$ is a k-strictly pseudo-contractive mapping on $C$, then the fixed point set $F(T)$ is closed convex, so that the projection $P_{F(T)}$ is well defined.

Lemma 2.2 [15]. Let $H$ be a Hilbert space and $C$ be a closed convex subset of $H$. Let $T: C \rightarrow H$ be a k-strictly pseudo-contractive mapping with $F(T) \neq \varnothing$. Then $F$ $\left(P_{C} \mathrm{~T}\right)=F(T)$.

Lemma 2.3 [15]. Let $H$ be a Hilbert space, $C$ be a closed convex subset of $H$, and $T$ : $C \rightarrow H$ be a k-strictly pseudo-contractive mapping. Define a mapping $S: C \rightarrow H$ by $S x=\lambda x+(1-\lambda)$ Tx for all $x \in C$. Then, as $\lambda \in[k, 1), S$ is a nonexpansive mapping such that $F(S)=F(T)$.

The following Lemmas 2.4 and 2.5 can be obtained from the Proposition 2.6 of Acedo and $\mathrm{Xu}[4]$. 
Lemma 2.4. Let $H$ be a Hilbert space and $C$ be a closed convex subset of $H$. For any $N \geq 1$, assume that for each $1 \leq i \leq N, T_{i}: C \rightarrow H$ is a $k_{i}$-strictly pseudo-contractive mapping for some $0 \leq k_{i}<1$. Assume that $\left\{\eta_{i}\right\}_{i=1}^{N}$ is a positive sequence such that $\sum_{i=1}^{N} \eta_{i}=1$. Then $\sum_{i=1}^{N} \eta_{i} T_{i}$ is a nonself- $k$-strictly pseudo-contractive mapping with $k=$ $\max \left\{k_{i}: 1 \leq i \leq N\right\}$.

Lemma 2.5. Let $\left\{T_{i}\right\}_{i=1}^{N}$ and $\left\{\eta_{i}\right\}_{i=1}^{N}$ be given as in Lemma 2.4. Suppose that $\left\{T_{i}\right\}_{i=1}^{N}$ has a common fixed point in $C$. Then $F\left(\sum_{i=1}^{N} \eta_{i} T_{i}\right)=\bigcap_{i=1}^{N} F\left(T_{i}\right)$.

Lemma $2.6[16,17]$. Let $\left\{s_{n}\right\}$ be a sequence of non-negative real numbers satisfying

$$
s_{n+1} \leq\left(1-\lambda_{n}\right) s_{n}+\lambda_{n} \delta_{n}+r_{n}, \quad n \geq 0,
$$

where $\left\{\lambda_{n}\right\},\left\{\delta_{n}\right\}$ and $\left\{r_{n}\right\}$ satisfy the following conditions:

(i) $\left\{\lambda_{n}\right\} \subset[0,1]$ and $\sum_{n=0}^{\infty} \lambda_{n}=\infty$,

(ii) $\lim \sup _{n \rightarrow \infty} \delta_{n} \leq 0$ or $\sum_{n=0}^{\infty} \lambda_{n} \delta_{n}<\infty$,

(iii) $r_{n} \geq 0(n \geq 0), \sum_{n=0}^{\infty} r_{n}=\infty$.

Then $\lim _{n \rightarrow \infty} s_{n}=0$.

Lemma 2.7 [18]. Let $\left\{x_{n}\right\}$ and $\left\{z_{n}\right\}$ be bounded sequences in a Banach space E and $\left\{\gamma_{n}\right\}$ be a sequence in $[0,1]$ which satisfies the following condition:

$$
0<\liminf _{n \rightarrow \infty} \gamma_{n} \leq \limsup _{n \rightarrow \infty} \gamma_{n}<1 .
$$

Suppose that $x_{n+1}=\gamma_{n} x_{n}+\left(1-\gamma_{n}\right) z_{n}$ for all $n \geq 0$ and

$$
\limsup _{n \rightarrow \infty}\left(\left\|z_{n+1}-z_{n}\right\|-\left\|x_{n+1}-x_{n}\right\|\right) \leq 0 .
$$

Then $\lim _{n \rightarrow \infty}|| z_{n}-x_{n} \|=0$.

Lemma 2.8. In a Hilbert space $H$, the following inequality holds:

$$
\|x+y\|^{2} \leq\|x\|^{2}+2\langle y, x+y\rangle, \quad x, y \in H .
$$

Lemma 2.9. Let $C$ be a nonempty closed convex subset of a Hilbert space $H$ such that $C \pm C \subset C$. Let $F: C \rightarrow C$ be a $\kappa$-Lipschitzian and $\eta$-strongly monotone operator with $\kappa>0$ and $\eta>0$. Let $0<\mu<\frac{2 \eta}{\kappa^{2}}$ and $0<t<\rho<1$. Then $S:=\rho I-t \mu F: C \rightarrow C$ is a contraction with contractive constant $\rho-t \tau$, where $\tau=\frac{1}{2} \mu\left(2 \eta-\mu \kappa^{2}\right)<1$ with $t<\frac{1}{\tau}$.

Proof. From (1.3), (1.4) and (2.1), we have

$$
\begin{aligned}
\|S x-S y\|^{2} & =\|\rho(x-y)-t \mu(F x-F y)\|^{2} \\
& =\rho^{2}\|x-y\|^{2}+t^{2} \mu^{2}\|F x-F y\|^{2}-2 t \rho \mu\langle F x-F y, x-y\rangle \\
& \leq \rho^{2}\|x-y\|^{2}+t^{2} \mu^{2} \kappa^{2}\|x-y\|-2 t \rho \mu \eta\|x-y\|^{2} \\
& <\rho^{2}\|x-y\|^{2}+t \rho \mu^{2} \kappa^{2}\|x-y\|-2 t \rho \mu \eta\|x-y\|^{2} \\
& =\left(\rho^{2}-t \rho \mu\left(2 \eta-\mu \kappa^{2}\right)\right)\|x-y\|^{2} \\
& <(\rho-t \tau)^{2}\|x-y\|^{2}
\end{aligned}
$$

where $\tau=\frac{1}{2} \mu\left(2 \eta-\mu \kappa^{2}\right)<1$, and so

$$
\|S x-S y\|<(\rho-t \tau)\|x-y\| .
$$

Hence $S$ is a contraction with contractive constant $\rho-t \tau$. $\square$

\section{Main results}

We need the following result for the existence of solutions of a certain variational inequality, which is slightly an improvement of Theorem 3.1 of Tian [12]. 
Theorem T2. Let $H$ be a Hilbert space, $C$ be a closed convex subset of $H$ such that $C \pm$ $C \subset C$, and $T: C \rightarrow C$ be a nonexpansive mapping with $F(T) \neq \varnothing$. Let $F: C \rightarrow C$ be a $\kappa$-Lipschitzian and $\eta$-strongly monotone operator with $\kappa>0$ and $\eta>0$. Let $f: C \rightarrow C$ be a contraction with the contractive constant $\alpha \in(0,1)$. Let $0<\mu<\frac{2 \eta}{\kappa^{2}}$, $0<\gamma<\frac{\mu\left(\eta-\frac{\mu \kappa^{2}}{2}\right)}{\alpha}=\frac{\tau}{\alpha}$ and $\tau<1$. Let $x_{t}$ be a fixed point of a contraction St $\ni x \alpha$ tyf $(x)+$ $(I-t \mu F) T x$ for $t \in(0,1)$ and $t<\frac{1}{\tau}$. Then $\left\{x_{t}\right\}$ converges strongly to a fixed point $\tilde{x}$ of $T$ as $t \rightarrow 0$, which solves the following variational inequality:

$$
\langle\mu F \tilde{x}-\gamma f(\tilde{x}), \tilde{x}-p\rangle \leq 0, \quad p \in F(T) .
$$

Equivalently, we have $P_{F(T)}(I-\mu F+\gamma f) \tilde{x}=\tilde{x}$.

Now, we study the strong convergence result for a general iterative scheme (IS).

Theorem 3.1. Let $H$ be a Hilbert space, $C$ be a closed convex subset of $H$ such that $C \pm$ $C \subset C$, and $T: C \rightarrow H$ be a k-strictly pseudo-contractive mapping with $F(T) \neq \varnothing$ for some $0 \leq k<1$. Let $F: C \rightarrow C$ be a $\kappa$-Lipschitzian and $\eta$-strongly monotone operator with $\kappa>0$ and $\eta>0$. Let $f: C \rightarrow C$ be a contraction with the contractive constant $\alpha \in(0,1)$. Let $0<\mu<\frac{2 \eta}{\kappa^{2}}, 0<\gamma<\frac{\mu\left(\eta-\frac{\mu \kappa^{2}}{2}\right)}{\alpha}=\frac{\tau}{\alpha}$ and $\tau<1$. Let $\left\{\left\{\alpha_{n}\right\}\right.$ and $\left\{\beta_{n}\right\}$ be sequences in $(0,1)$ which satisfy the conditions:

(C1) $\lim _{n \rightarrow \infty} \alpha_{n}=0$;

(C2) $\Sigma_{n=0}^{\infty} \alpha_{n}=\infty$;

(B) $0<\lim \inf _{n \rightarrow \infty} \beta_{n} \leq \lim \sup _{n \rightarrow \infty} \beta n<1$.

Let $\left\{x_{n}\right\}$ be a sequence in $C$ generated by

$$
\left\{\begin{array}{l}
x_{0} \in C, \\
x_{n+1}=\alpha_{n} \gamma f\left(x_{n}\right)+\beta_{n} x_{n}+\left(\left(1-\beta_{n}\right) I-\alpha_{n} \mu F\right) P_{C} S x_{n}, \quad n \geq 0,
\end{array}\right.
$$

where $S: C \rightarrow H$ is a mapping defined by $S x=k x+(1-k) T x$ and $P_{C}$ is the metric projection of $H$ onto $C$. Then $\left\{x_{n}\right\}$ converges strongly to $q \in F(T)$, which solves the following variational inequality:

$$
\langle\mu F q-\gamma f(q), q-p\rangle \leq 0, \quad p \in F(T) .
$$

Proof. First, from the condition (C1), without loss of generality, we assume that $\alpha_{n} \tau$ $<1, \frac{2 \alpha_{n}(\tau-\gamma \alpha)}{1-\alpha_{n} \alpha \gamma}<1$ and $\alpha_{n}<\left(1-\beta_{n}\right)$ for $n \geq 0$.

We divide the proof several steps:

Step 1. We show that $\left\|x_{n}-p\right\| \leq \max \left\{\left\|x_{0}-p\right\|, \frac{\|\gamma f(p)-\mu F p\|}{\tau-\gamma \alpha}\right\}$ for all $n \geq 0$ and all $p$ $\in F(T)=F(S)$. Indeed, let $p \in F(T)$. Then from Lemma 2.9, we have

$$
\begin{aligned}
\left\|x_{n+1}-p\right\|= & \| \alpha_{n}\left(\gamma f\left(x_{n}\right)-\mu F p\right)+\beta_{n}\left(x_{n}-p\right) \\
& \quad+\left(\left(1-\beta_{n}\right) I-\alpha_{n} \mu F\right) P_{C} S x_{n}-\left(\left(1-\beta_{n}\right) I-\alpha_{n} \mu F\right) P_{C} S p \| \\
\leq & \left(1-\beta_{n}-\alpha_{n} \tau\right)\left\|x_{n}-p\right\|+\beta_{n}\left\|x_{n}-p\right\|+\alpha_{n}\left\|\gamma f\left(x_{n}\right)-\mu F p\right\| \\
\leq & \left(1-\alpha_{n} \tau\right)\left\|x_{n}-p\right\|+\alpha_{n}\left(\left\|\gamma f\left(x_{n}\right)-\gamma f(p)\right\|+\|\gamma f(p)-\mu F p\|\right) \\
\leq & \left(1-(\tau-\gamma \alpha) \alpha_{n}\right)\left\|x_{n}-p\right\|+(\tau-\gamma \alpha) \alpha_{n} \frac{\|\gamma f(p)-\mu F p\|}{\tau-\gamma \alpha} \\
\leq & \max \left\{\left\|x_{n}-p\right\|, \frac{\|\gamma f(p)-\mu F p\|}{\tau-\gamma \alpha}\right\} .
\end{aligned}
$$


Using an induction, we have $\left\|x_{n}-p\right\| \leq \max \left\{\left\|x_{0}-p\right\|, \frac{\|\gamma f(p)-\mu F p\|}{\tau-\gamma \alpha}\right\}$. Hence, $\left\{x_{n}\right\}$ is bounded, and so are $\left\{f\left(x_{n}\right)\right\},\left\{P_{C} S x_{n}\right\}$ and $\left\{F P_{C} S x_{n}\right\}$.

Step 2. We show that $\lim _{n \rightarrow \infty}|| x_{n+1}-x_{n} \|=0$. To this show, define

$$
x_{n+1}=\beta_{n} x_{n}+\left(1-\beta_{n}\right) z_{n,} \text { for all } n \geq 0 .
$$

Observe that from the definition of $z_{n}$,

$$
\begin{aligned}
& z_{n+1}-z_{n} \\
= & \frac{x_{n+2}-\beta_{n+1} x_{n+1}}{1-\beta_{n+1}}-\frac{x_{n+1}-\beta_{n} x_{n}}{1-\beta_{n}} \\
= & \frac{\alpha_{n+1} \gamma f\left(x_{n+1}\right)+\left(\left(1-\beta_{n+1}\right) I-\alpha_{n+1} \mu F\right) P_{C} S x_{n+1}}{1-\beta_{n+1}} \\
& -\frac{\alpha_{n} \gamma f\left(x_{n}\right)+\left(\left(1-\beta_{n}\right) I-\alpha_{n} \mu F\right) P_{C} S x_{n}}{1-\beta_{n}} \\
= & \frac{\alpha_{n+1}}{1-\beta_{n+1}} \gamma f\left(x_{n+1}\right)-\frac{\alpha_{n}}{1-\beta_{n}} \gamma f\left(x_{n}\right) \\
& +P_{C} S x_{n+1}-P_{C} S x_{n}+\frac{\alpha_{n}}{1-\beta_{n}} \mu F P_{C} S x_{n}-\frac{\alpha_{n+1}}{1-\beta_{n+1}} \mu F P_{C} S x_{n+1} \\
= & \frac{\alpha_{n+1}}{1-\beta_{n+1}}\left(\gamma f\left(x_{n+1}\right)-\mu F P_{C} S x_{n+1}\right) \\
& +\frac{\alpha_{n}}{1-\beta_{n}}\left(\mu F P_{C} S x_{n}-\gamma f\left(x_{n}\right)\right)+P_{C} S x_{n+1}-P_{C} S x_{n} .
\end{aligned}
$$

Thus, it follows that

$$
\begin{aligned}
\left\|z_{n+1}-z_{n}\right\|-\left\|x_{n+1}-x_{n}\right\| \leq & \frac{\alpha_{n+1}}{1-\beta_{n+1}}\left(\gamma\left\|f\left(x_{n+1}\right)\right\|+\mu\left\|F P_{C} S x_{n+1}\right\|\right) \\
& +\frac{\alpha_{n}}{1-\beta_{n}}\left(\mu\left\|F P_{C} S x_{n}\right\|+\gamma\left\|f\left(x_{n}\right)\right\|\right) .
\end{aligned}
$$

From the condition (C1) and (B), it follows that

$$
\limsup _{n \rightarrow \infty}\left(\left\|z_{n+1}-z_{n}\right\|-\left\|x_{n+1}-x_{n}\right\|\right) \leq 0 .
$$

Hence, by Lemma 2.7, we have

$$
\lim _{n \rightarrow \infty}\left\|z_{n}-x_{n}\right\|=0 .
$$

Consequently,

$$
\lim _{n \rightarrow \infty}|| x_{n+1}-x_{n}\left\|=\lim _{n \rightarrow \infty}\left(1-\beta_{n}\right)\right\| z_{n}-x_{n} \|=0 .
$$

Step 3. We show that $\lim _{n \rightarrow \infty}|| x_{n}-P_{C} S x_{n} \|=0$. Indeed, since

$$
x_{n+1}=\alpha_{n} \gamma f\left(x_{n}\right)+\beta_{n} x_{n}+\left(\left(1-\beta_{n}\right) I-\alpha_{n} \mu F\right) P_{C} S x_{n},
$$

we have

$$
\begin{aligned}
\left\|x_{n}-P_{C} S x_{n}\right\| \leq & \left\|x_{n}-x_{n+1}\right\|+\left\|x_{n+1}-P_{C} S x_{n}\right\| \\
\leq & \left\|x_{n}-x_{n+1}\right\|+\alpha_{n}\left\|\gamma f\left(x_{n}\right)-\mu F P_{C} S x_{n}\right\| \\
& +\beta_{n}\left\|x_{n}-P_{C} S x_{n}\right\|,
\end{aligned}
$$

that is,

$$
\left\|x_{n}-P_{C} S x_{n}\right\| \leq \frac{1}{1-\beta_{n}}\left\|x_{n}-x_{n+1}\right\|+\frac{\alpha_{n}}{1-\beta_{n}}\left\|\gamma f\left(x_{n}\right)-\mu F P_{C} S x_{n}\right\| .
$$


So, from the conditions (C1) and (B) and Step 2, it follows that

$$
\lim _{n \rightarrow \infty}\left\|x_{n}-P_{C} S x_{n}\right\|=0 .
$$

Step 4. We show that

$$
\limsup _{n \rightarrow \infty}\left\langle\gamma f(q)-\mu F q, x_{n}-q\right\rangle \leq 0,
$$

where $q=\lim _{t \rightarrow 0} x_{t}$ being $x_{t}=t \gamma f\left(x_{t}\right)+(I-t \mu F) P_{C} S x_{t}$ for $0<t<1$ and $t<\frac{1}{\tau}$. We note that from Lemmas 2.2 and 2.3 and Theorem T2, $q \in F(T)=F(S)$ and $q$ is a solution of a variational inequality

$$
\langle\mu F q-\gamma f(q), q-p\rangle \leq 0, \quad p \in F(T) .
$$

To show this, we can choose a subsequence $\left\{x_{n_{j}}\right\}$ of $\left\{x_{n}\right\}$ such that

$$
\lim _{j \rightarrow \infty}\left\langle\gamma f(q)-\mu F q, \quad x_{n_{j}}-q\right\rangle=\limsup _{n \rightarrow \infty}\left\langle\gamma f(q)-\mu F q, x_{n}-q\right\rangle .
$$

Since $\left\{x_{n}\right\}$ is bounded, there exists a subsequence $\left\{x_{n_{j_{i}}}\right\}$ of $\left\{x_{n_{j}}\right\}$ which converges weakly to $w$. Without loss of generality, we can assume that $x_{n_{j}} \rightarrow w$. Since $\left\|x_{n}-P_{C} S x_{n}\right\| \rightarrow 0$ by Step 3, we obtain $w=P_{C} S w$. In fact, if $w \neq P_{C} S w$, then, by Opial condition,

$$
\begin{aligned}
\liminf _{j \rightarrow \infty}\left\|x_{n_{j}}-w\right\| & <\liminf _{j \rightarrow \infty}\left\|x_{n_{j}}-P_{C} S w\right\| \\
& \leq \liminf _{j \rightarrow \infty}\left(\left\|x_{n_{j}}-P_{C} S x_{n_{j}}\right\|+\left\|P_{C} S x_{n_{j}}-P_{C} S w\right\|\right) \\
& \leq \liminf _{j \rightarrow \infty}\left\|x_{n_{j}}-w\right\|,
\end{aligned}
$$

which is a contradiction. Hence $w=P_{C} S w$. Since $F\left(P_{C} S\right)=F(S)$, from Lemma 2.3, we have $w \in F(T)$. Therefore, from (3.1), we conclude that

$$
\begin{aligned}
\limsup _{n \rightarrow \infty}\left\langle\gamma f(q)-\mu F q, x_{n}-q\right\rangle & =\lim _{j \rightarrow \infty}\left\langle\gamma f(q)-\mu F q, x_{n_{j}}-q\right\rangle \\
& =\langle\gamma f(q)-\mu F q, w-q\rangle \\
& \leq 0 .
\end{aligned}
$$

Step 5. We show that $\lim _{n \rightarrow \infty}|| x_{n}-q \|=0$, where $q=\lim _{t \rightarrow 0} x_{t}$ being $x_{t}=t \gamma f(x t)+$ $(I-t \mu F) P_{C} S x_{t}$ for $0<t<1$ and $t<\frac{1}{\tau}$, and $q$ is a solution of a variational inequality

$$
\langle\mu F q-\gamma f(q), q-p\rangle \leq 0, \quad p \in F(T) .
$$

Indeed, from (IS), we have

$$
\begin{aligned}
x_{n+1}-q= & \alpha_{n}\left(\gamma f\left(x_{n}\right)-\mu F q\right)+\beta_{n}\left(x_{n}-q\right) \\
& +\left(\left(1-\beta_{n}\right) I-\alpha_{n} \mu F\right) P_{C} S x_{n}-\left(\left(1-\beta_{n}\right) I-\alpha_{n} \mu F\right) q .
\end{aligned}
$$

Applying Lemmas 2.8 and 2.9, we have

$$
\begin{aligned}
& \left\|x_{n+1}-q\right\|^{2} \\
\leq & \left\|\beta_{n}\left(x_{n}-q\right)+\left(\left(1-\beta_{n}\right) I-\alpha_{n} \mu F\right) P_{C} S x_{n}-\left(\left(1-\beta_{n}\right) I-\alpha_{n} \mu F\right) P_{C} S q\right\|^{2} \\
& +2 \alpha_{n}\left\langle\gamma f\left(x_{n}\right)-\mu F q, x_{n+1}-q\right\rangle \\
\leq & \left(\left(1-\beta_{n}-\alpha_{n} \tau\right)\left\|x_{n}-q\right\|+\beta_{n}\left\|x_{n}-q\right\|\right)^{2} \\
& +2 \alpha_{n} \gamma\left\langle f\left(x_{n}\right)-f(q), x_{n+1}-q\right\rangle+2 \alpha_{n}\left\langle\gamma f(q)-\mu F q, x_{n+1}-q\right\rangle \\
\leq & \left(1-\tau \alpha_{n}\right)^{2}\left\|x_{n}-q\right\|^{2}+2 \alpha_{n} \gamma \alpha\left\|x_{n}-q\right\|\left\|x_{n+1}-q\right\| \\
& +2 \alpha_{n}\left\langle\gamma f(q)-\mu F q, x_{n+1}-q\right\rangle \\
\leq & \left(1-\tau \alpha_{n}\right)^{2}\left\|x_{n}-q\right\|^{2}+\alpha_{n} \gamma \alpha\left(\left\|x_{n}-q\right\|^{2}+\left\|x_{n+1}-q\right\|^{2}\right) \\
& +2 \alpha_{n}\left\langle\gamma f(q)-\mu F q, x_{n+1}-q\right\rangle,
\end{aligned}
$$


that is,

$$
\begin{aligned}
\left\|x_{n+1}-q\right\|^{2} \leq & \frac{1-2 \tau \alpha_{n}+\tau^{2} \alpha_{n}^{2}+\alpha_{n} \gamma \alpha}{1-\alpha_{n} \gamma \alpha}\left\|x_{n}-q\right\|^{2} \\
& +\frac{2 \alpha_{n}}{1-\alpha_{n} \gamma \alpha}\left\langle\gamma f(q)-\mu F q, x_{n+1}-q\right\rangle \\
= & \left(1-\frac{2(\tau-\gamma \alpha) \alpha_{n}}{1-\alpha_{n} \gamma \alpha}\right)\left\|x_{n}-q\right\|^{2}+\frac{\tau^{2} \alpha_{n}^{2}}{1-\alpha_{n} \gamma \alpha}\left\|x_{n}-q\right\|^{2} \\
& +\frac{2 \alpha_{n}}{1-\alpha_{n} \gamma \alpha}\left\langle\gamma f(q)-\mu F q, x_{n+1}-q\right\rangle \\
\leq & \left(1-\frac{2(\tau-\gamma \alpha)}{1-\alpha_{n} \gamma \alpha} \alpha_{n}\right)\left\|x_{n}-q\right\|^{2}+\frac{2(\tau-\gamma \alpha) \alpha_{n}}{1-\alpha_{n} \gamma \alpha} \times \\
= & \left(1-\lambda_{n}\right)\left\|x_{n}-q\right\|^{2}+\lambda_{n} \delta_{n},
\end{aligned}
$$

where $M=\sup \left\{\left\|x_{n}-q\right\| 2: n \geq 0\right\}, \lambda_{n}=\frac{2(\tau-\gamma \alpha)}{1-\alpha_{n} \gamma \alpha} \alpha_{n}$ and

$$
\delta_{n}=\frac{\tau^{2} \alpha_{n}}{2(\tau-\gamma \alpha)} M+\frac{1}{\tau-\gamma \alpha}\left\langle\gamma f(q)-\mu F q, x_{n+1}-q\right\rangle .
$$

From the conditions (C1) and (C2) and Step 4, it is easy to see that $\lambda_{n} \rightarrow 0$, $\sum_{n=0}^{\infty} \lambda_{n}=\infty$, and $\lim \sup _{n \rightarrow \infty} \delta_{n} \leq 0$. Hence, by Lemma 2.7 , we conclude $x_{n} \rightarrow q$ as $n \rightarrow \infty$. This completes the proof.

Remark 3.1. (1) Theorem 3.1 extends and develops Theorem 3.2 of Tian [12] from a nonexpansive mapping to a strictly pseudo-contractive mapping together with removing the condition (C3) $\Sigma_{n=0}^{\infty}\left|\alpha_{n+1}-\alpha_{n}\right|<\infty$.

(2) Theorem 3.1 also generalizes Theorem 2.1 of Jung [6] as well as Theorem 2.1 of Cho et al. [5] and Theorem 3.4 of Marino and Xu [10] from a strongly positive bounded linear operator $A$ to a $\kappa$-Lipschitzian and $\eta$-strongly monotone operator $F$.

(3) Theorem 3.1 also improves the corresponding results of Halpern [13], Moudafi [8], Wittmann [14] and $\mathrm{Xu}$ [9] as some special cases.

Theorem 3.2. Let $H$ be a Hilbert space, $C$ be a closed convex subset of $H$ such that $C \pm C \subset C$, and $T_{i}: C \rightarrow H$ be a $k_{i}$-strictly pseudo-contractive mapping for some $0 \leq k_{i}$ $<1$ and $\bigcap_{i=1}^{N} F\left(T_{i}\right) \neq \emptyset$. Let $F: C \rightarrow C$ be a $\kappa$-Lipschitzian and $\eta$-strongly monotone operator with $\kappa>0$ and $\eta>0$. Let $f: C \rightarrow C$ be a contraction with the contractive constant $\alpha \in(0,1)$. Let $0<\mu<\frac{2 \eta}{\kappa^{2}}, 0<\gamma<\frac{\mu\left(\eta-\frac{\mu \kappa^{2}}{2}\right)}{\alpha}=\frac{\tau}{\alpha}$ and $\tau<1$. Let $\left\{\alpha_{n}\right\}$ and $\{\beta n\}$ be sequences in $(0,1)$ which satisfy the conditions.

(C1) $\lim _{n \rightarrow \infty} \alpha_{n}=0$;

(C2) $\Sigma_{n=0}^{\infty} \alpha_{n}=\infty$;

(B) $0<\liminf _{n \rightarrow \infty} \beta_{n} \leq \lim \sup _{n \rightarrow \infty} \beta_{n}<1$.

Let $\left\{x_{n}\right\}$ be a sequence in $C$ generated by

$$
\left\{\begin{array}{l}
x_{0} \in C, \\
x_{n+1}=\alpha_{n} \gamma f\left(x_{n}\right)+\beta_{n} x_{n}+\left(\left(1-\beta_{n}\right) I-\alpha_{n} \mu F\right) P_{C} S x_{n}, \quad n \geq 0,
\end{array}\right.
$$

where $S: C \rightarrow H$ is a mapping defined by $S x=k x+(1-k) \sum_{i=1}^{N} \eta_{i} T_{i} x$ with $k=\max \left\{k_{i}\right.$ $: 1 \leq i \leq N\}$ and $\left\{\eta_{i}\right\}$ is a positive sequence such that $\sum_{i=1}^{N} \eta_{i}=1$ and $P_{C}$ is the metric 
projection of $H$ onto $C$. Then $\left\{x_{n}\right\}$ converges strongly to $q \in F(T)$, which solves the following variational inequality:

$$
\langle\mu F q-\gamma f(q), q-p\rangle \leq 0, \quad p \in \bigcap_{i=1}^{N} F\left(T_{i}\right) .
$$

Proof. Define a mapping $T: C \rightarrow H$ by $T x=\sum_{i=1}^{N} \eta_{i} T_{i} x$. By Lemmas 2.4 and 2.5, we conclude that $T: C \rightarrow H$ is a $k$-strictly pseudo-contractive mapping with $k=\max \left\{k_{i}\right.$ : $1 \leq i \leq N\}$ and $F(T)=F\left(\sum_{i=1}^{N} \eta_{i} T_{i}\right)=\bigcap_{i=1}^{N} F\left(T_{i}\right)$. Then the result follows from Theorem 3.1 immediately.

As a direct consequence of Theorem 3.2, we have the following result for nonexpansive mappings (that is, 0 -strictly pseudo-contractive mappings).

Theorem 3.3. Let $H$ be a Hilbert space, $C$ be a closed convex subset of $H$ such that $C \pm C \subset C,\left\{T_{i}\right\}_{i=1}^{N}: C \rightarrow$ Hbe a finite family of nonexpansive mappings with $\bigcap_{i=1}^{N} F\left(T_{i}\right) \neq \emptyset$. Let $F: C \rightarrow C$ be a $\kappa$-Lipschitzian and $\eta$-strongly monotone operator with $\kappa>0$ and $\eta>0$. Let $f: C \rightarrow C$ be a contraction with the contractive constant $\alpha \in$ $(0,1)$. Let $0<\mu<\frac{2 \eta}{\kappa^{2}}, 0<\gamma<\frac{\mu\left(\eta-\frac{\mu \kappa^{2}}{2}\right)}{\alpha}=\frac{\tau}{\alpha}$ and $\tau<1$. Let $\left\{\alpha_{n}\right\}$ and $\{\beta n\}$ be sequences in $(0,1)$ which satisfy the conditions.

(C1) $\lim _{n \rightarrow \infty} \alpha_{n}=0$;

(C2) $\Sigma_{n=0}^{\infty} \alpha_{n}=\infty$;

(B) $0<\lim \inf _{n \rightarrow \infty} \beta_{n} \leq \lim \sup _{n \rightarrow \infty} \beta_{n}<1$.

Let $\left\{x_{n}\right\}$ be a sequence in $C$ generated by

$$
\left\{\begin{array}{l}
x_{0} \in C, \\
x_{n+1}=\alpha_{n} \gamma f\left(x_{n}\right)+\beta_{n} x_{n}+\left(\left(1-\beta_{n}\right) I-\alpha_{n} \mu F\right) P_{C} \sum_{i=1}^{N} \eta_{i} T_{i} x_{n}, \quad n \geq 0
\end{array}\right.
$$

where $\left\{\eta_{i}\right\}_{i=1}^{N}$ is a positive sequence such that $\sum_{i=1}^{N} \eta_{i}=1$ and $P_{C}$ is the metric projection of $H$ onto $C$. Then $\left\{x_{n}\right\}$ converges strongly to a common fixed point $q$ of $\left\{T_{i}\right\}_{i=1}^{N}$, which solves the following variational inequality:

$$
\langle\mu F q-\gamma f(q), q-p\rangle \leq 0, \quad p \in \bigcap_{i=1}^{N} F\left(T_{i}\right) .
$$

Remark 3.2. (1) Theorems 3.2 and 3.3 also generalize Theorems 2.2 and 2.4 of Jung [6] from a strongly positive bounded linear operator $A$ to a $\kappa$-Lipschitzian and $\eta$ strongly monotone operator $F$.

(2) Theorems 3.2 and 3.3 also improve and complement the corresponding results of Cho et al. [5] by removing the condition (C3) $\Sigma_{n=0}^{\infty}\left|\alpha_{n+1}-\alpha_{n}\right|<\infty$ together with using a $\kappa$-Lipschitzian and $\eta$-strongly monotone operator $F$.

(3) As in [19], we also can establish the result for a countable family $\left\{T_{i}\right\}$ of $k_{i}$-strict pseudo-contractive mappings with $0 \leq k_{i}<1$.

\section{Competing interests}

The authors declare that they have no competing interests. 
References

1. Browder, FE: Fixed point theorems for noncompact mappings. Proc Natl Acad Sci USA. 53, 1272-1276 (1965). doi:10.1073/pnas.53.6.1272

2. Browder, FE: Convergence of approximants to fixed points of nonexpansive nonlinear mappings in Banach spaces. Arch Ration Mech Anal. 24, 82-90 (1967)

3. Browder, FE, Petryshn, W: Construction of fixed points of nonlinear mappings Hilbert space. J Math Anal Appl. 20, 197-228 (1967). doi:10.1016/0022-247X(67)90085-6

4. Acedo, GL, Xu, HK: Iterative methods for strictly pseudo-contractions in Hilbert space. Nonlinear Anal. 67, 2258-2271 (2007). doi:10.1016/j.na.2006.08.036

5. Cho, YJ, Kang, SM, Qin, X: Some results on k-strictly pseudo-contractive mappings in Hilbert spaces. Nonlinear Anal. 70 , 1956-1964 (2009). doi:10.1016/j.na.2008.02.094

6. Jung, JS: Strong convergence of iterative methods for k-strictly pseudo-contractive mappings in Hilbert spaces. Appl Math Comput. 215, 3746-3753 (2010). doi:10.1016/j.amc.2009.11.015

7. Morales, $\mathrm{CH}$, Jung, JS: Convergence of paths for pseudo-contractive mappings in Banach spaces. Proc Am Math Soc. 128, 3411-3419 (2000). doi:10.1090/50002-9939-00-05573-8

8. Moudafi, A: Viscosity approximation methods for fixed-points problems. J Math Anal Appl. 241, 46-55 (2000). doi:10.1006/jmaa.1999.6615

9. Xu, HK: Viscosity approximation methods for nonexpansive mappings. J Math Anal Appl. 298, 279-291 (2004). doi:10.1016/j.jmaa.2004.04.059

10. Marino, G, Xu, HX: A general iterative method for nonexpansive mappings in Hilbert spaces. J Math Anal Appl. 318, 43-52 (2006). doi:10.1016/j.jmaa.2005.05.028

11. Yamada, I: The hybrid steepest descent for the variational inequality problems over the intersection of fixed points sets of nonexpansive mappings. In: Butnariu D, Censor Y, Reich S (eds.) Inherently Parallel Algorithms in Feasibility and Optimization and Their Applications. pp. 473-504. Elservier, New York (2001)

12. Tian, M: A general itewrative algorithm for nonexpansive mappings in Hilbert spaces. Nonlinear Anal. 73, 689-694 (2010). doi:10.1016/j.na.2010.03.058

13. Halpern, B: Fixed points of nonexpansive maps. Bull Am Math Soc. 73, 957-961 (1967). doi:10.1090/S0002-9904-1967$11864-0$

14. Wittmann, R: Approximation of fixed points of nonexpansive mappings. Arch Math. 58, 486-491 (1992). doi:10.1007/ BF01190119

15. Zhou, $\mathrm{H}$ : Convergence theorems of fixed points for $k$-strict pseudo-contractions in Hilbert spaces. Nonlinear Anal. 69 , 456-462 (2008). doi:10.1016/j.na.2007.05.032

16. Liu, LS: Iterative processes with errors for nonlinear strongly accretive mappings in Banach spaces. J Math Anal Appl. 194, 114-125 (1995). doi:10.1006/jmaa.1995.1289

17. Xu, HK: Iterative algorithms for nonlinear operators. J Lond Math Soc. 66, 240-256 (2002). doi:10.1112/ S0024610702003332

18. Suzuki, T: Strong convergence of Krasnoselskii and Mann's type sequences for one parameter nonexpansive semigroups without Bochner integral. J Math Anal Appl. 305, 227-239 (2005). doi:10.1016/j.jmaa.2004.11.017

19. Aoyama, K, Kimura, Y, Takahashi, W, Toyoda, M: Approximation of common fixed points of a countable family of nonexpansive mappings in a Banach space. Nonlinear Anal. 67, 2350-2360 (2007). doi:10.1016/j.na.2006.08.032

doi:10.1186/1687-1812-2011-24

Cite this article as: Jung: Some results on a general iterative method for $k$-strictly pseudo-contractive mappings.

Fixed Point Theory and Applications 2011 2011:24.

\section{Submit your manuscript to a SpringerOpen ${ }^{\circ}$ journal and benefit from:}

- Convenient online submission

Rigorous peer review

- Immediate publication on acceptance

- Open access: articles freely available online

- High visibility within the field

- Retaining the copyright to your article

Submit your next manuscript at $\boldsymbol{s p r i n g e r o p e n . c o m ~}$ 